\title{
Revealing Majorana fermion states in a superfluid of cold atoms subject to a harmonic potential
}

\author{
Tao Zhou ${ }^{1,2}$ and Z. D. Wang ${ }^{2}$ \\ ${ }^{1}$ College of Science, Nanjing University of Aeronautics and Astronautics, Nanjing 210016, China \\ ${ }^{2}$ Department of Physics and Center of Theoretical and Computational Physics, The University of Hong Kong, \\ Pokfulam Road, Hong Kong, China
}

(Received 19 September 2012; published 14 October 2013)

\begin{abstract}
We here explore Majorana fermion states in an s-wave superfluid of cold atoms in the presence of spin-orbital coupling and an additional harmonic potential. The superfluid boundary is induced by a harmonic trap. Two locally separated Majorana fermion states are revealed numerically based on the self-consistent Bogoliubov-de Gennes equations. The local density of states is calculated, through which the signatures of Majorana excitations may be indicated experimentally.
\end{abstract}

DOI: 10.1103/PhysRevB.88.155114

PACS number(s): 71.10.Pm, 03.67.Lx, 74.90.+n

\section{INTRODUCTION}

The Majorana fermion (MF), a particle that is its own antiparticle, was originally proposed by Majorana many years ago. ${ }^{1}$ For the past decade, the MFs have attracted tremendous attention due to their exotic properties. Especially manipulating MFs is one promising way to realize the non-Abelian statistics that may have potential applications in topological quantum computation. $^{2}$ For condensed matter systems, the MFs were first predicted to emerge in the Pfaffian fractional quantum Hall state. ${ }^{3}$ Later, it was proposed that the MFs exist in the $p+i p$ superconducting system associated with the zeroenergy bound states in the vortex core. ${ }^{4-6}$ While searching for the spin-triplet $p$-wave superconducting materials is of a great challenge. Besides the real material, the $p+i p$ superfluid (SF) pairing state was proposed to be artificially created in the cold atom systems with the $p$-wave Feshbach resonance. ${ }^{6}$ However, $p$-wave molecules are not stable so that the proposal is also difficult to implement. ${ }^{7}$ Recently, more attention has been paid to topological systems with the spin-orbital interaction. ${ }^{8-17}$ This kind of system with an $s$-wave pairing may be equivalent to the $p+i p$ superconductor. ${ }^{8-11}$ Experimentally, tremendous efforts have been made to search for MFs. Very recently, several groups have reported the signatures of MFs in various systems, ${ }^{18-22}$ but a definite demonstration for the existence of MFs is still awaited.

It has been indicated that both the spin-orbital coupling and the $s$-wave pairing SF state can be artificially created in cold atom systems. The spin orbital coupling can be generated through spatially varying laser fields. ${ }^{14,23,24}$ The $s$-wave pairing state is much more stable than the $p$-wave one and has been realized. ${ }^{25}$ Besides the above conditions, the realization of MFs require the presence of the Zeeman field and vortex. A feasible scheme to realize a controllable Zeeman field in cold atoms was proposed in Ref. 14. In the present work, instead of the realization of MFs in the vortex core, we elaborate that the MF states may also be induced in a confined harmonic potential, noting that the position and motion of the confined potential may be controlled more easily in the cold atoms. Based on the Bogoliubov-de Gennes (BdG) equations, the SF order parameters are calculated self-consistently. Our numerical results illustrate the existence of the MF states. The distinct features of the MF states are discussed in detail.
The article is organized as follows. In Sec. II, we introduce the model and work out the formalism. In Sec. III, we perform numerical calculations and discuss the obtained results. Finally, we give a brief summary in Sec. IV.

\section{HAMILTONIAN AND FORMALISM}

We start from a Hamiltonian including the spin-orbital coupling and the SF pairing term, which reads

$$
H=H_{t}+H_{\Delta} .
$$

Here, $H_{t}$ includes the tight-binding part of the model with the spin-orbital interaction and the spin polarization term given by $^{26}$

$$
\begin{aligned}
H_{t}= & \sum_{i}\left(\psi_{i}^{\dagger} \frac{\sigma_{3}-i \sigma_{1}}{2} \psi_{i+\hat{x}}+\psi_{i}^{\dagger} \frac{\sigma_{3}-i \sigma_{2}}{2} \psi_{i+\hat{y}}+\text { H.c. }\right) \\
& +\sum_{i} \psi_{i}^{\dagger}\left(U_{i} \sigma_{0}+h \sigma_{3}\right) \psi_{i}
\end{aligned}
$$

with $\psi_{i}=\left(\psi_{i \uparrow}, \psi_{i \downarrow}\right)^{\mathrm{T}}$, where $\sigma_{n}$ are the identity $(n=0)$ and Pauli matrix $(n=1,2,3)$, respectively, $h$ represents an effective Zeeman field. While $H_{\Delta}$ is the SF pairing term, expressed as

$$
H_{\Delta}=\sum_{i}\left(\Delta_{i} \psi_{i}^{\dagger} i \sigma_{2} \psi_{i}^{\dagger}+\text { H.c. }\right) .
$$

The above Hamiltonian can be diagonalized by solving the BdG equations self-consistently,

$$
\left(\begin{array}{cc}
H_{t}(\mathbf{r}) & \Delta(\mathbf{r}) \sigma_{3} \\
\Delta^{*}(\mathbf{r}) \sigma_{3} & -\sigma_{2} H_{t}^{*}(\mathbf{r}) \sigma_{2}
\end{array}\right) \Psi^{n}(\mathbf{r})=E_{n} \Psi^{n}(\mathbf{r}) .
$$

For the $N \times N$ lattice size, the dimensionality of the Hamiltonian matrix is $4 N \times 4 N . \Psi^{n}(\mathbf{r})$ is a $4 N$ order column vector with $\Psi^{n}(\mathbf{r})=\left(u_{\mathbf{r} \uparrow}^{n}, u_{\mathbf{r} \downarrow}^{n}, v_{\mathbf{r} \downarrow}^{n}, v_{\mathbf{r} \uparrow}^{n}\right)^{\mathrm{T}}$.

The order parameters $\Delta(\mathbf{r})$ are determined selfconsistently:

$$
\Delta(\mathbf{r})=\frac{V}{2} \sum_{n} u_{\mathbf{r} \uparrow}^{n} v_{\mathbf{r} \downarrow}^{n *} \tanh \left(\frac{E_{n}}{2 K_{B} T}\right),
$$

with $V$ the pairing strength. 
The on-site particle number with spin $\sigma$ is calculated after diagonalizing the BdG Hamiltonian,

$$
\left\langle n_{i \sigma}\right\rangle=\sum_{n}\left|u_{i \sigma}^{n}\right|^{2} f\left(E_{n}\right),
$$

where $f(x)$ is the Fermi distribution function. Then we can obtain the on-site particle number $n_{i}=\left\langle n_{i \uparrow}\right\rangle+\left\langle n_{i \downarrow}\right\rangle$ and the site-dependent magnetization $m_{i}=1 / 2\left(\left\langle n_{i \uparrow}\right\rangle-\left\langle n_{i \downarrow}\right\rangle\right)$, respectively. The local density of states (LDOS) can be calculated as

$$
\rho_{i}(\omega)=\sum_{n}\left[\left|u_{i \uparrow}^{n}\right|^{2} \delta\left(E_{n}-\omega\right)+\left|v_{i \downarrow}^{n}\right|^{2} \delta\left(E_{n}+\omega\right)\right] .
$$

The $\delta$ function $\delta(x)$ is taken as $\delta=\Gamma /\left[\pi\left(x^{2}+\Gamma^{2}\right)\right]$, with the quasiparticle damping $\Gamma=0.01$.

We first analyze theoretically the duality between the present model with the $p$-wave superconductors. For the uniform case with $U_{i}=0$, the Hamiltonian can be expressed in the momentum space as

$$
H=H_{t \mathbf{k}}+\sum_{\mathbf{k}}\left(\Delta_{\mathbf{k}} e^{i \phi_{\mathbf{k}}} \psi_{\mathbf{k}, \uparrow} \psi_{-\mathbf{k}, \downarrow}+\text { H.c. }\right),
$$

where $H_{t \mathbf{k}}$ is the tight-banding term given by

$$
\begin{aligned}
H_{t \mathbf{k}}= & \sum_{\mathbf{k}} h(\mathbf{k})\left(\psi_{\mathbf{k} \uparrow}^{\dagger} \psi_{\mathbf{k} \uparrow}-\psi_{\mathbf{k} \downarrow}^{\dagger} \psi_{\mathbf{k} \downarrow}\right) \\
& +\sum_{\mathbf{k}}\left[g(\mathbf{k}) \psi_{\mathbf{k} \uparrow}^{\dagger} \psi_{\mathbf{k} \downarrow}+\text { H.c. }\right],
\end{aligned}
$$

with $h(\mathbf{k})=\left(m+\cos k_{x}+\cos k_{y}\right) \quad$ and $\quad g(\mathbf{k})=\sin k_{x}+$ $i \sin k_{y}$. Defining two spinless quasiparticle operators $c_{\mathbf{k}}=\frac{1}{\sqrt{2}}\left(\psi_{\mathbf{k} \uparrow}^{\dagger}+e^{i \phi_{\mathbf{k}}} \psi_{-\mathbf{k} \downarrow}\right)$ and $d_{\mathbf{k}}=\frac{i}{\sqrt{2}}\left(\psi_{\mathbf{k} \uparrow}^{\dagger}-e^{i \phi_{\mathbf{k}}} \psi_{-\mathbf{k} \downarrow}\right)$, the Hamiltonian can be rewritten in the spinless representation:

$$
\begin{aligned}
H= & \sum_{\mathbf{k}} \Delta_{\mathbf{k}} C^{\dagger}(\mathbf{k}) \sigma_{0} C(\mathbf{k})+\sum_{\mathbf{k}} h(\mathbf{k}) C^{\dagger}(\mathbf{k}) \sigma_{3} C(\mathbf{k}) \\
& +\sum_{\mathbf{k}}\left[g(\mathbf{k}) e^{i \phi_{\mathbf{k}}} C^{\dagger}(\mathbf{k}) C^{\dagger}(-\mathbf{k})+\text { H.c. }\right],
\end{aligned}
$$

with $C(\mathbf{k})=\left[(d(\mathbf{k}), c(\mathbf{k})]^{\mathrm{T}}\right.$. One can find that the spin-orbital interaction is transformed to the pairing terms. The system is equivalent to the $p+i p$ system with two sets of quasiparticles, respectively, expressed as $c$ and $\underset{\sim}{d}$ operators. The pairing terms for both sets have the form $\widetilde{\Delta}=\sin k_{x}+i \sin k_{y}$. This transformation makes it possible to realize the MFs in the spin-orbital system.

The $H_{t}$ in Eq. (2) with $U_{i}=0$ is an effective model to describe the topological insulator. ${ }^{26}$ The topological properties are determined by $h$ with the nonzero Chern number for $0<$ $|h|<2$. Figure 1(a) illustrates the chiral edge states of the model by considering the open boundary condition along $x$ direction $\left(L_{x}=50\right)$ and periodic boundary condition along $y$ direction with $h=-0.2$. As is seen, the energy gap closes at $k_{y}=\pi$ with the zero-energy mode being localized at the edge $\left(i_{x}=0,50\right)$, suggesting the existence of the edge state. On the other hand, if periodic boundary condition is taken for both directions, the Hamiltonian can be expressed as $2 \times 2$ matrix in the momentum space [see Eq. (9)]. The two energy bands, obtained by diagonalizing the above $2 \times 2$ matrix, are plotted in Fig. 1(b), representing the energy spectrum in the bulk.

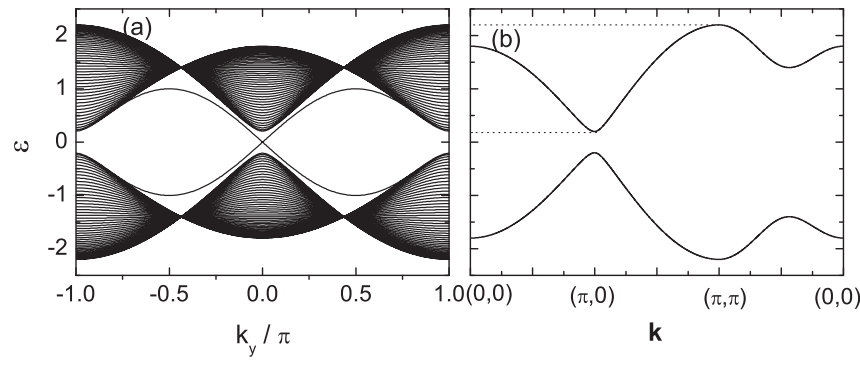

FIG. 1. (a) The energy spectrum of the Hamiltonian Eq. (2) with an open boundary condition along the $x$ direction. (b) The energy band in the momentum space with periodic condition.

There are two bands with the gap 0.4 and the energy bandwidth $2(\varepsilon \ni[-2.2,-0.2] \cup[0.2,2.2])$. If the on-site potential $U_{i}$ with $0.2<U_{i}<2.2$ is chosen, the Fermi energy would cross the lower energy band and the system becomes metal-like. Then, the SF pairing might occur when an additional pairing potential term is taken into account.

\section{RESULTS AND DISCUSSION}

The presence of the vortex is likely a key ingredient but a great challenge for probing the MFs and non-Abelian statistics. Usually, the vortex is induced by the magnetic field, characterized as a $2 \pi$ winding of the phase and the vanishing of the order parameter in its center. In the present work, we will not consider the field-induced vortex, we rather take into account an harmonic potential in the form of $U_{i}=U_{0}\left|\left(\mathbf{r}_{\mathbf{i}}-\mathbf{r}_{\mathbf{0}}\right)\right|^{2}$. We can conclude from the band structure shown in Fig. 1(b) that the SF order parameter is nearly zero in the trap center and increases when away from the center. It will reach the maximum value when the potential $U_{i}$ crosses the energy band. Two SF boundaries are expected to exist when the on-site potential crosses the band edges. The MF excitation may appear at the SF boundaries.

Now, let us illustrate numerically the existence of the MF states based on the above proposal. In the following, we use $h=-0.2, U_{0}=0.01$ and the pairing potential $V=2$. The numerical calculation is performed on a $36 \times 36$ square lattice with the periodic boundary condition. The trapping center locates at $\mathbf{r}_{\mathbf{0}}=(18,18)$.

The self-consistent results of the $\mathrm{BdG}$ equations are presented in Fig. 2. Figure 2(a) displays the spatial distribution of the gap magnitudes and their phases (denoted by arrows). The spatial distribution of the particle number and the sitedependent magnetization are presented in Figs. 2(b) and 2(c), respectively. The two-dimensional cuts of the gap magnitude, the particle number, and the site dependent magnetism are plotted in Fig. 2(d). As is seen, the gap is nearly zero at the trap center and increases when it is away from the center. It reaches the maxima at about $\left|\mathbf{r}_{\mathbf{i}}-\mathbf{r}_{\mathbf{0}}\right|=11$. Then, the gap decreases and becomes nearly zero at the border. The SF region forms a ring with the gap magnitudes being nearly isotropic around the ring. The phases of the SF order parameters change from 0 to $2 \pi$ around the ring. Thus the self-consistent results for the order parameters are somewhat similar to the case of the vortex state for superconducting materials in the magnetic field. Actually, here the presence of the phase winding is due 

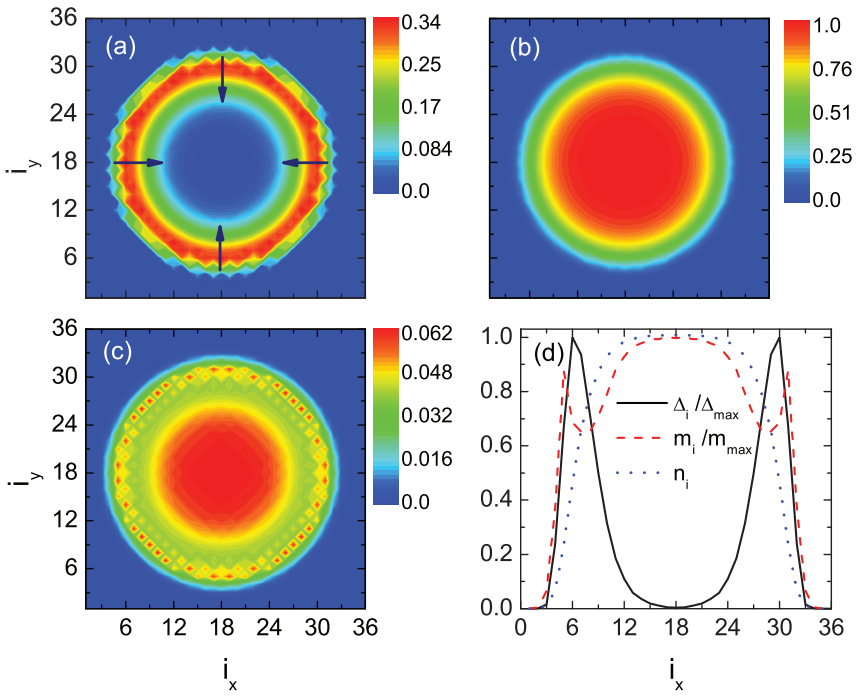

FIG. 2. (Color online) (a) The numerical results for the magnitudes and phases (shown by arrows) of the pairing order parameters. (b) and (c) are the particle number and magnetism, respectively. (d) The two-dimensional cut of the pairing magnitude, particle number, and magnetism along $i_{y}=18$, respectively.

to the presence of the Zeeman field, which is also an effective vertical magnetic field.

The competition between the SF and ferromagnetism is elucidated in Figs. 2(b) and 2(d), which is a key point for the zero mode discussed below. When $\left|\mathbf{r}_{\mathbf{i}}-\mathbf{r}_{\mathbf{0}}\right|<4.6\left(U_{i}<0.2\right)$, the on-site energy is smaller than the minimum excitation energy, and thus the system behaves as a ferromagnetic insulator with the particle number per site being fixed to near 1.0. The magnetization reaches a maximum and the pairing order parameter is negligibly small. As $4.6<\left|\mathbf{r}_{\mathbf{i}}-\mathbf{r}_{\mathbf{0}}\right|<15$ $\left(0.2<U_{i}<2.2\right)$, the Fermi energy crosses the energy band. Therefore the lower band becomes unfilled, so that the average on-site particle number and the ferromagnetic order decrease. As a result, the SF order shows up. We can also see that the evolution of ferromagnetism is nonmonotonous, namely, it reaches the minimum as the pairing order is of maximum and recover to a local maxima value as the pairing order decreases, indicating the competition between SF order and ferromagnetism. As $\left|\mathbf{r}_{\mathbf{i}}-\mathbf{r}_{\mathbf{0}}\right|>15$, the on-site energy is larger than the maximum-excited energy of the band. Both the particle number and the order parameters decrease to zero.

As presented in Fig. 2, when the on-site energy is small or very large, the particle number is fixed to be 1 or 0 , and thus an insulating gap should exist. The insulating gap disappears when the on-site energy crosses the energy band. The system becomes metal-like and the SF pairing gap shows up. We can expect two effective insulator-SF boundaries inside and outside the SF ring. The zero mode is expected to exist at the boundaries. We diagonalize the Hamiltonian and all of the 5184 eigenvalues are plotted in Fig. 3. As is seen, the eigenvalues are nearly continuous but a kink occurs at the zero energy. Enlarging the low-energy part in the inset (a) of Fig. 3, the eigenvalues are discontinuous. Intriguingly, our numerical calculation reveals a zero-energy fermionic mode. This zero-energy mode is protected by an energy gap about $\Delta_{p}=0.027$. We also checked numerically the robustness of

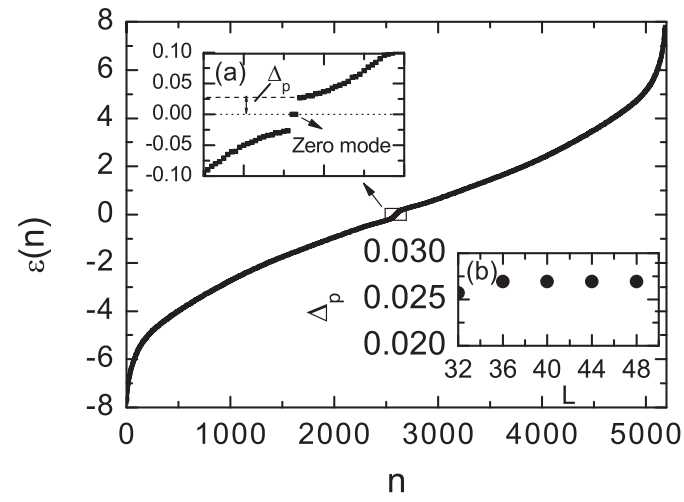

FIG. 3. The eigenvalues for the Hamiltonian matrix. Inset: (a) the replot of the rectangle part in the main figure. (b) The minimum nonzero eigenvalues $\Delta_{p}$ vs the system size $L$.

this result. The gap $\Delta_{p}$ as a function of the system size is plotted in the inset (b) of Fig. 3. As is illustrated, the gap is almost unchanged as the system's size increases from 36 sites, saturating seemingly to 0.027 . Thus the zero mode, formed near the gap edge, is robust and can hardly be excited by local perturbations, which is consistent with the previous analytical analysis for $p$-wave superconductors. ${ }^{4}$

As is known, the creation/annihilation operator of the captioned zero mode is self-Hermitian, ${ }^{4}$ namely, there may be two MFs associated with this zero mode, with the corresponding operators being given by $\gamma_{1}=\left(C+C^{\dagger}\right) / \sqrt{2}$ and $\gamma_{2}=$ $i\left(C^{\dagger}-C\right) / \sqrt{2}$, where $C^{\dagger}=\sum_{i \sigma}\left(u_{i \sigma} \psi_{i \sigma}^{\dagger}+v_{i \sigma} \psi_{i \sigma}\right)$, which can be obtained from the zero-energy eigenvector. In this sense, we are able to study numerically the MF states by solving the $\mathrm{BdG}$ equations. The spatial distribution of the two MF states $\rho_{1,2}$ with the units of $\rho_{\max }$ are presented in Figs. 4(a) and 4(b), respectively. Since two MFs at the same location
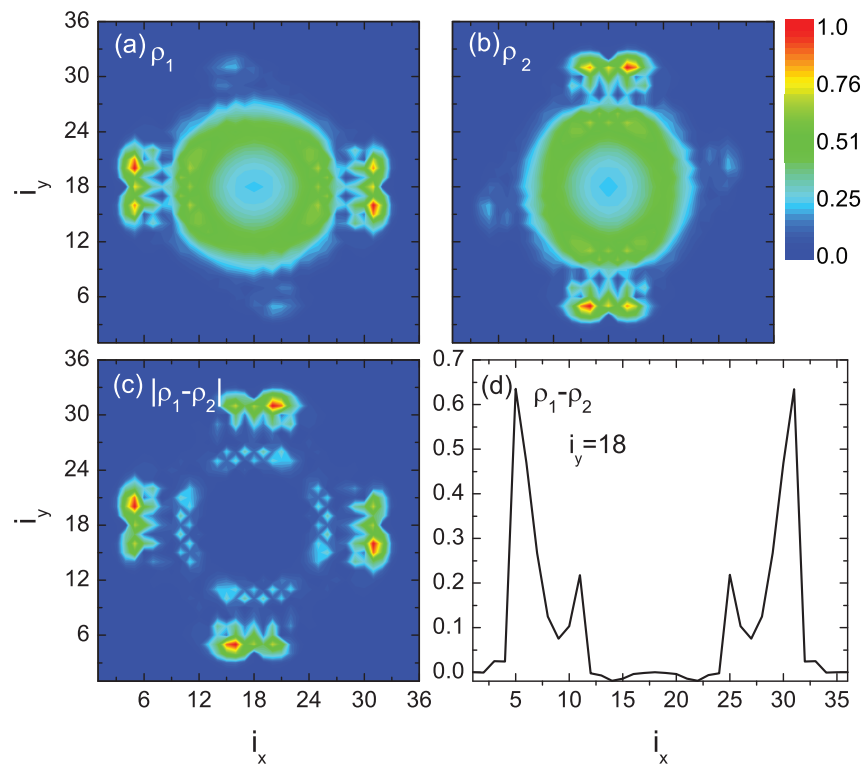

FIG. 4. (Color online) The intensity plot of the spatial distributions of (a) $\gamma_{1}$ and (b) $\gamma_{2}$. (c) The difference of the probability distributions between $\gamma_{1}$ and $\gamma_{2}$. (d) The two-dimensional cut of $\rho_{1}-\rho_{2}$ along $i_{y}=18$. 

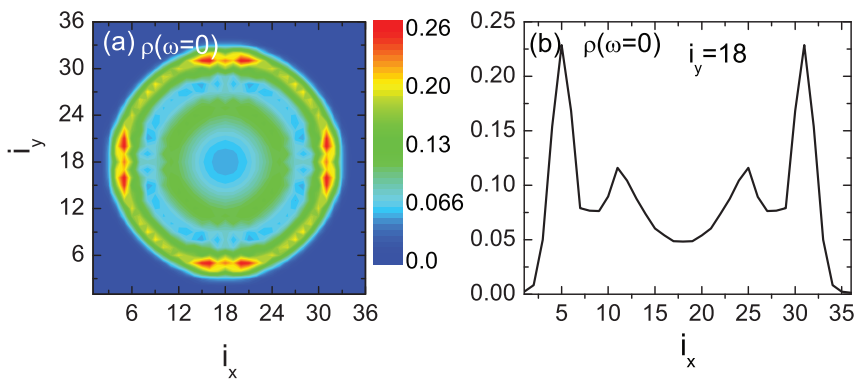

FIG. 5. (Color online) (a) The intensity plot of the zero-energy LDOS. (b) The two-dimensional cut of the zero-energy LDOS.

shall annihilate to an ordinary fermion, only locally separated MFs are meaningful. It should be insightful to eliminate the overlap part of the two MF states by looking into the difference of the two MFs $\left(\left|\rho_{1}-\rho_{2}\right|\right)$, as presented in Fig. 4(c). The two-dimensional cut of $\rho_{1}-\rho_{2}$ along $i_{y}=18$ is plotted in Fig. 4(d).

The two separated MF states may be identified from the results presented in Fig. 4. They appear at the SF-insulator boundaries with maximum probabilities being at the horizontal (vertical) directions for $\gamma_{1}\left(\gamma_{2}\right)$. One significant feature revealed by Fig. 4 is the nonlocal behavior for both states. The MF states are symmetric about the trapping center, and thus both of them should have two equivalent maxima. Inside the SF ring, there exists a certain extent of overlap between these two states; while around outsider boundary, there is almost no overlap between the two states. This overlap behavior is a distinct feature and would not occur for the usual MFs in the well-separated vortex cores. As is known, for the usual vortex bounded MF, the wave function decreases exponentially away from the vortex core. For the present case, the overlap of the MF states seems more interesting and may be controlled by the trap potential.

It is also insightful to look into the LDOS for investigating the existence and distribution of the above zero mode. The intensity plot of the zero-energy LDOS spectra is plotted in Fig. 5(a). Its two-dimensional cut for $i_{y}=18$ is plotted in Fig. 5(b). Generally, the zero-energy LDOS could describe the excitations of low-energy quasiparticle fermions. Due to the existence of the quasiparticle damping, it is contributed not only the exact zero-energy quasiparticles, but also some low-energy fermionic states. For the present case, since there exists an energy gap between the zero-energy state and the lowest excited state, as is shown in Fig. 3, the contributions from nonzero energy state should be rather small. As a result, here the LDOS spectra may be qualitatively consistent with the distributions of the two MF states. This point could be seen by comparing Figs. 4 and 5. Especially, from the two-dimensional cut of the spectra shown in Figs. 4(d) and 5(b), our results indicate that the zero-energy LDOS are qualitatively the same as the results of $\rho_{1}-\rho_{2}$. Therefore the indication of MF modes may be provided through observing experimentally the LDOS spectra. This may establish a useful link for theoretical analysis and experimental observations.

Finally, we wish to address the significance and possible applications of the present work. First, we have developed a way to realize the exotic MF states. As seen from Fig. 4, the overlap of two MF states $\gamma_{1}$ and $\gamma_{2}$ occurs inside the SF ring (with the relatively small amplitude), while they are well separated at the outside boundary. In the MF state space, this kind of overlap may be treated as an effective interaction between the two MFs $\gamma_{1}$ and $\gamma_{2}$. This interaction may be quite small in comparison with the gap and thus should be unable to change the "quasimode" nature of the MFs, particularly for a realistic large size system. In this sense, these quasimode MFs can still exhibit the non-Abelian statistics feature and thus may have potential applications in topological quantum computing. Secondly, the MF state is subjected to the harmonic potential. Technically, the position and strength of harmonic potentials can be well controlled, and thus one might manipulate the MF states through operating the potential. Thirdly, the well-controlled MFs may be used to demonstrate the non-Abelian statistics through their couplings to a "quantum dot," as proposed in Ref. 27. At last, it is of fundamental interest to explore possible new states with cold atoms. It was proposed that merons with a half elementary charge may be realized in small systems. ${ }^{28,29}$ Since there exist two degrees of freedom for the spin triplet pairing, it is quite intriguing to look for or probe merons in a similar $p$-wave system or topological superconducting system, and to compare the properties of merons with those of MF modes.

\section{SUMMARY}

In summary, we have proposed and elaborated a scenario to realize the MF states in fermionic cold atom systems. The numerical results have shown that the SF region forms a ring with the MF states being mainly around the boundaries of the ring. It is rather promising that the presence of MF zero modes may be detected experimentally through the LDOS spectra.

\section{ACKNOWLEDGMENTS}

We thank Q. H. Wang and S. L. Zhu for helpful discussions. This work was supported by the NCET (Grant No. NCET-120626), the NSFC (Grant No. 11374005), the GRF (Grants No. HKU7058/11P and No. HKU7045/13P), and the CRF (Grant No. HKU8/11G) of Hong Kong.
${ }^{1}$ E. Majorana, Il Nuovo Cimento 14, 171 (1937).

${ }^{2}$ C. Nayak, S. H. Simon, A. Stern, M. Freedman, and S. Das Sarma, Rev. Mod. Phys. 80, 1083 (2008).

${ }^{3}$ G. Moore and N. Read, Nucl. Phys. B 360, 362 (1991).

${ }^{4}$ N. Read and D. Green, Phys. Rev. B 61, 10267 (2000).

${ }^{5}$ D. A. Ivanov, Phys. Rev. Lett. 86, 268 (2001).
${ }^{6}$ S. Tewari, S. Das Sarma, C. Nayak, C. Zhang, and P. Zoller, Phys. Rev. Lett. 98, 010506 (2007).

${ }^{7}$ Y. Inada, M. Horikoshi, S. Nakajima, M. Kuwata-Gonokami, M. Ueda, and T. Mukaiyama, Phys. Rev. Lett. 101, 100401 (2008).

${ }^{8}$ L. Fu and C. L. Kane, Phys. Rev. Lett. 100, 096407 (2008). 
${ }^{9}$ M. Sato, Y. Takahashi, and S. Fujimoto, Phys. Rev. Lett. 103, 020401 (2009).

${ }^{10}$ C. Zhang, S. Tewari, R. M. Lutchyn, and S. Das Sarma, Phys. Rev. Lett. 101, 160401 (2008).

${ }^{11}$ J. Liu, Q. Han, L. B. Shao, and Z. D. Wang, Phys. Rev. Lett. 107, 026405 (2011).

${ }^{12}$ J. Linder, Y. Tanaka, T. Yokoyama, A. Sudb $\varnothing$, and N. Nagaosa, Phys. Rev. Lett. 104, 067001 (2010).

${ }^{13}$ J. D. Sau, S. Tewari, R. M. Lutchyn, T. D. Stanescu, and S. Das Sarma, Phys. Rev. B 82, 214509 (2010).

${ }^{14}$ S.-L. Zhu, L.-B. Shao, Z. D. Wang, and L.-M. Duan, Phys. Rev. Lett. 106, 100404 (2011).

${ }^{15}$ X.-J. Liu, L. Jiang, H. Pu, and H. Hu, Phys. Rev. A 85, 021603(R) (2012).

${ }^{16}$ L. Jiang, T. Kitagawa, J. Alicea, A. R. Akhmerov, D. Pekker, G. Refael, J. I. Cirac, E. Demler, M. D. Lukin, and P. Zoller, Phys. Rev. Lett. 106, 220402 (2011).

${ }^{17}$ Y. X. Zhao and Z. D. Wang, arXiv:1305.3791v2; arXiv:1305.1251v2; Phys. Rev. Lett. 110, 240404 (2013).

${ }^{18}$ J. R. Williams, A. J. Bestwick, P. Gallagher, S. S. Hong, Y. Cui, A. S. Bleich, J. G. Analytis, I. R. Fisher, and D. Goldhaber-Gordon, Phys. Rev. Lett. 109, 056803 (2012).

${ }^{19}$ L. P. Rokhinson, X. Liu, and J. K. Furdyna, Nat. Phys. 8, 795 (2012).

${ }^{20}$ M. T. Deng, C. L. Yu, G. Y. Huang, M. Larsson, P. Caroff, and H. Q. Xu, Nano Lett. 12, 6414 (2012).
${ }^{21}$ A. Das, Y. Ronen, Y. Most, Y. Oreg, M. Heiblum, and H. Shtrikman, Nat. Phys. 8, 887 (2012).

${ }^{22}$ V. Mourik, K. Zuo, S. M. Frolov, S. R. Plissard, E. P. A. M. Bakkers, and L. P. Kouwenhoven, Science 336, 1003 (2012).

${ }^{23}$ G. Juzeliunas and P. Ohberg, Phys. Rev. Lett. 93, 033602 (2004); J. Ruseckas, G. Juzeliūnas, P. Öhberg, and M. Fleischhauer, ibid. 95, 010404 (2005); P. Zhang, Y. Li, and C. P. Sun, Eur. Phys. J. D 36, 229 (2005).

${ }^{24}$ S. L. Zhu, H. Fu, C. J. Wu, S. C. Zhang, and L. M. Duan, Phys. Rev. Lett. 97, 240401 (2006); X.-J. Liu, X. Liu, L. C. Kwek, and C. H. Oh, ibid. 98, 026602 (2007); T. D. Stanescu, B. Anderson, and V. Galitski, Phys. Rev. A 78, 023616 (2008); C. Zhang, ibid. 82, 021607(R) (2010).

${ }^{25}$ T. Bourdel, L. Khaykovich, J. Cubizolles, J. Zhang, F. Chevy, M. Teichmann, L. Tarruell, S. J. J. M. F. Kokkelmans, and C. Salomon, Phys. Rev. Lett. 93, 050401 (2004); J. K. Chin, D. E. Miller, Y. Liu, C. Stan, W. Setiawan, C. Sanner, K. Xu, and W. Ketterle, Nature (London) 443, 961 (2006).

${ }^{26}$ X. L. Qi, Y. S. Wu, and S. C. Zhang, Phys. Rev. B 74, 045125 (2006); X.-L. Qi, T. L. Hughes, and S.-C. Zhang, ibid. 78, 195424 (2008).

${ }^{27}$ K. Flensberg, Phys. Rev. Lett. 106, 090503 (2011).

${ }^{28}$ M. V. Milovanovic, E. Dobardzic, and Z. Radovic, Phys. Rev. B 80, 125305 (2009).

${ }^{29}$ A. Petkovic and M. V. Milovanovic, Phys. Rev. Lett. 98, 066808 (2007). 